\section{Tests and Analysis for SLC Damping Ring Data*}

J.E. Spencer

Stanford Linear Accelerator Center, Stanford University

Stanford, California 94309
SLAC-PUB-6089

April 1993

(A/I)
Abstract: Beyond typical, open-loop or feedback use, fast, turn-by-turn bunch monitors provide recursive data that determines system dimensionality $d$ and $\mathrm{K}$-entropy whose time fluctuations provide useful diagnostic tools. For injection and extraction, one can study variations in the dimensionality of the attractor with matching, jitter, current and tunes. Comparison to dynamical models then helps to optimize control. Experimental and theoretical examples and their signatures are discussed e.g. the evolution of the distribution function after injection is studied as a solution of Liouville's equation.

\section{Introduction}

Optimal injection and extraction in storage rings can be approached in several ways. We can try to understand the problem with an explicit Hamiltonian or in a purely heuristic way using feedback/forward or, as argued earlier, with a closed system whose structure is based on dynamical models [1]. Since chaotic dynamics are controllable [2] with data of sufficient accuracy and sampling bandwidth, we study the information content in time series, how it varies with time and how we can control and use it.

\section{Tests of data}

For $N$ equi-spaced samples $\left\langle\overrightarrow{\boldsymbol{x}}_{i}(i \tau): i=1 \cdots N\right\rangle_{t}$, we can write the correlation function as:

$$
C(\rho)=\frac{2}{(N-n+1)^{2}} \sum_{j>i=1}^{N-n+1} \Theta\left(\rho-\left|\vec{x}_{i}-\vec{x}_{j}\right|\right)
$$

with $\rho$ the correlation length, $\Theta$ the Heaviside function and $n$ the number of variables assumed to span the problem [3]. $d$ is the limit of $\mathrm{d} \log C(\rho) / \mathrm{d} \log \rho$ with $n_{\max }>d>D$ where $D$ counts the number of degrees of freedom.

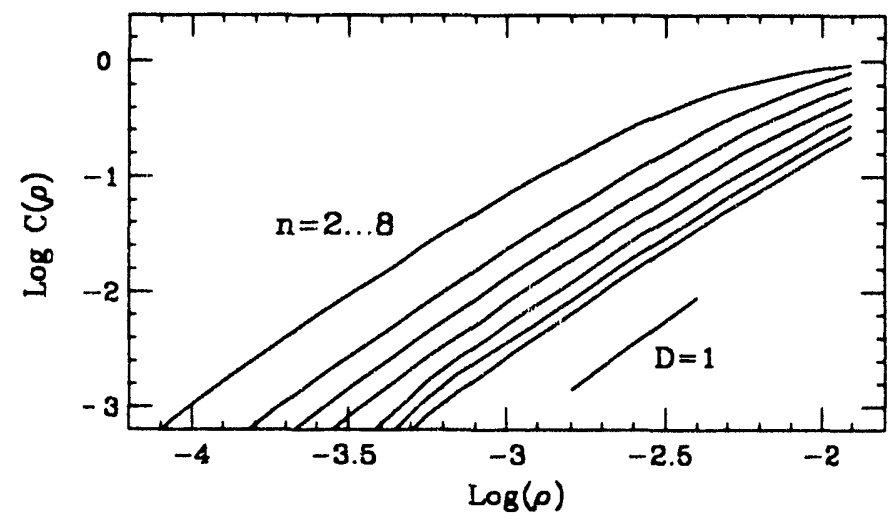

Fig. 1: Dependence of correlation integral on size.

* Work supported by DOE contract DE-AC03-76SF00515.
Fig. 1 shows the analysis for $\Delta x$ from Fig. 3. All curves in Fig. 3 give $D \approx 1$, including 1000 particle tracking. The collective motion is well represented by a single particle with $1 \mathrm{D}$. Two constraints on decimated 'data' are to avoid obvious correlations e.g. $n>5$ in Fig's. $1 \& 3$ and to have enough data to unfold deterministic and stochastic effects (large $d$ ). For contrast, Fig. 2 shows the expected result for 1000 random points. For $C(\rho) \ll 1$, all curves in Fig. 1 are parallel whereas none are in Fig. 2.

\section{Analytic model for injection}

Even when one reduces particle losses along a closed orbit at injection by adjusting the transverse tunes to avoid resonances, injection may still not be optimal due to the cumulative effect of nonlinear fields on the beam over many turns. In terms of the lowest order moments, one observes decoherence in the center-of-mass motion $\left\langle{ }^{2}\right\rangle_{t}$ and filamentation of the phase space e.g. growth in $\left\langle x^{2}\right\rangle_{t}$. Examples are given below: first from Moshammer's analytic model [4], then tracking and finally from measured SLC data.

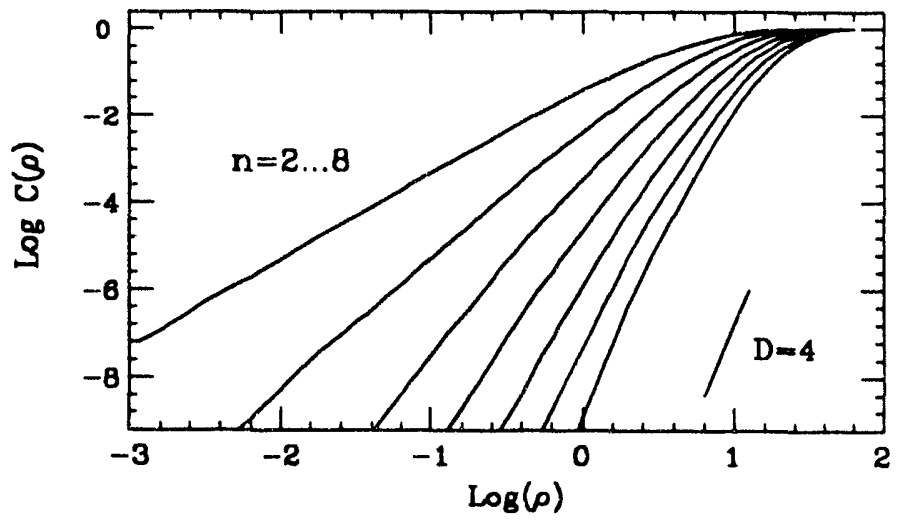

Fig. 2: Correlation data for 1000 random turns.

\section{A. Assumptions}

1. One can replace the Hamiltonian of single particle motion $H(x, p, s)$ by an Hamiltonian, averaged over the fast evolving variables

$$
\hat{H}(I)=\omega_{0} \sum_{n=1}^{N} \mu_{n-1} \frac{I^{n}}{n !} \text {. }
$$

The quantity $\omega_{0}$ denotes the revolution frequency times the tune. The linear motion is described with $\mu_{0}=1$ and $N=1$ and $\mu_{1}$ contains first order octupole plus second order sextupole contributions etc.

Once the Hamiltonian is transformed into an integrable Hamiltonian, the equation of motion and thus the time evolution of the phase space distribution is determinable 
2. The beam distribution at injection is assumed Gaussian.

The only requirement is that the initial distribution is well approximated by a positi :e definite, but not necessarily smooth, function of the phase space variables.

3. Normal coordinates $(\xi, \eta)$, related to the transverse betatron coordinates $\left(x_{\beta}, p_{\beta}\right)$ are used:

$$
\xi=\frac{x_{\beta}}{\sqrt{\beta}}, \quad \eta=\frac{\alpha x_{\beta}+\beta p_{\beta}}{\sqrt{\beta}}, \quad \eta+i \xi=\sqrt{2 I} e^{i \phi} .
$$

Their relation to action-angle variables is given by the transformation from cartesian to polar coordinates.

The corresponding distribution function in $(I, \phi)$ at $t=0$ is:

$$
\begin{aligned}
\Psi=\frac{1}{2 \pi \epsilon_{x 0}} & \exp \left(-\frac{b+c}{\epsilon_{x 0}}\left[\sqrt{I} \cos (\Omega)-\sqrt{I_{0}} \cos \left(\Omega_{0}\right)\right]^{2}\right. \\
& \left.-\frac{b-c}{\epsilon_{x 0}}\left[\sqrt{I} \sin (\Omega)-\sqrt{I_{0}} \sin \left(\Omega_{0}\right)\right]^{2}\right)
\end{aligned}
$$

with

$$
\begin{array}{r}
\Omega=\phi-\int_{0}^{t} \text { at } \partial \widehat{H}(I) / \partial I-\bar{\phi} \quad \Omega_{0}=\phi_{0}-\bar{\phi} \\
I_{0}=\frac{1}{2}\left(\eta_{0}^{2}+\xi_{0}^{2}\right), \quad \phi_{0}=\arctan \left(\xi_{0} / \eta_{0}\right)
\end{array}
$$

where the center-of-mass at injection is given by the coordinates $\eta_{0}, \xi_{0}$ and $\epsilon_{x 0}$ denotes the injected emittance. The coefficients $b$ and $c$ describe the injected beam ellif se in the lattice of the storage ring. They are composed of the Twiss parameters associated with the injection point in the storage ring $(\alpha, \beta)$ and the Twiss parameters that describe the injected beam ellipse $\alpha_{b}, \beta_{b}$. The distribution function in Eq. (3) will be an exact solution of Liouville's equation. From Ref. [4] we have

and $c, \bar{\phi}$ are given by

$$
b=\frac{1}{2}\left(\frac{\beta_{b}}{\beta}+\frac{\beta}{\beta_{b}}+\frac{\beta}{\beta_{b}}\left[\alpha_{b}-\frac{\beta_{b}}{\beta} \alpha\right]^{2}\right)
$$

$$
c e^{2 i \bar{\phi}}=\frac{\beta}{2 \beta_{b}}\left(\left[\frac{\beta_{b}}{\beta}-i\left(\alpha_{b}-\frac{\beta_{b}}{\beta} \alpha\right)\right]^{2}-1\right) .
$$

Notice that: $c^{2}=b^{2}-1$. For $b=1$, the initial distribution is described in phase space by circular contours centered around $I_{0}, \phi_{0}$. In this case the beam is said to be matched to the lattice. 'The parameter $b$ is known as the $\beta$-mismatch parameter which quantifies the increase of effective beam size after filamentation.

\section{B. First Moments}

It is possible to obtain a closed expression for the first and second moments if we limit $N=2$ for the linear and quadratic terms in Eq. (2). From Ref. [4], the result for the first moment from $\Psi$ in $\mathrm{Eq} .(3)$ is:

$$
\begin{aligned}
& \langle\eta+i \xi\rangle=\iint \Psi \sqrt{2 I} \exp (\phi) d I d \phi \\
& =\frac{A \sqrt{2 I_{0}\left(b^{2}-c^{2}\right)}}{\kappa^{2}\left(1-z^{2}\right)^{3 / 2}} e^{-I_{0}\left[b+c \cos \left(2 \Omega_{0}\right)\right] / \epsilon_{x 0}} e^{i\left(\omega_{0} t+\bar{\phi}+\tilde{\Omega}_{0}\right)} \\
& \quad \times\left(1-z e^{-2 i \tilde{\Omega}_{0}}\right) \exp \left(\lambda-\lambda z \cos \left(2 \tilde{\Omega}_{0}\right)\right)
\end{aligned}
$$

with

$$
\kappa=b-i\left(\omega_{0} \mu_{1} t \epsilon_{x 0}\right), \quad z=c / \kappa, \quad \lambda=\frac{A^{2} I_{0}}{\kappa\left(1-z^{2}\right) \epsilon_{x 0}}
$$

and

$\tan \left(\tilde{\Omega}_{0}\right)=\frac{b-c}{b+c} \tan \left(\Omega_{0}\right), A=\sqrt{b^{2}+c^{2}+2 c b \cos \left(2 \Omega_{0}\right)}$

Using the definitions of $A$ and $\tilde{\Omega}_{0}$ below Eq. (3) it is straightforward to show that $\mathrm{Eq}$. (4) fulfills the initial condition $\langle\eta+i \xi\rangle_{t=0}=\sqrt{2 I_{0}} \exp \left(i \phi_{0}\right)$. Since $|\kappa|$ increases with time, the asymptotic value of the first moment tends to zero: $\langle\eta+i \xi\rangle_{t \rightarrow \infty}=0$. In this context one talks about the decoherence of the center-of-mass motion. This is illustrated in Fig. 3. One thousand particles were tracked over 2000 revolutions in the lattice of the SLC damping ring which contains 72 permanent sextupoles. The stars in Fig. 3 describe the analytic solution based on Eq. (4) whereas the lighter solid line refers to tracking. There is a small but stable phase error.

Equation (4) reduces considerably if we assume a matched beam at injection with $b=1$. In this case Eq. (4) describes the decoherence of an initial Gaussian beam that has been deflected from the reference orbit.

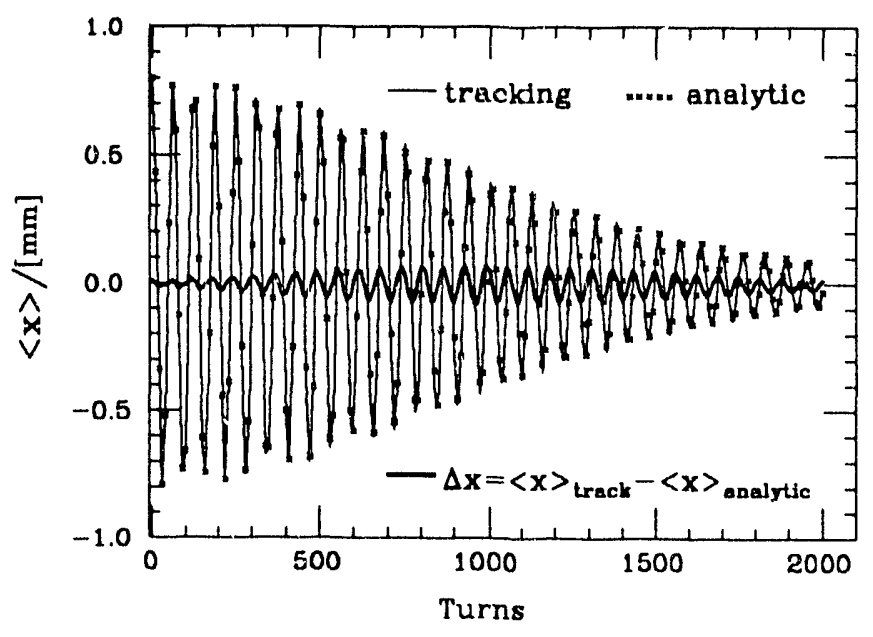

Fig. 3: Center-of-mass motion after injection. 


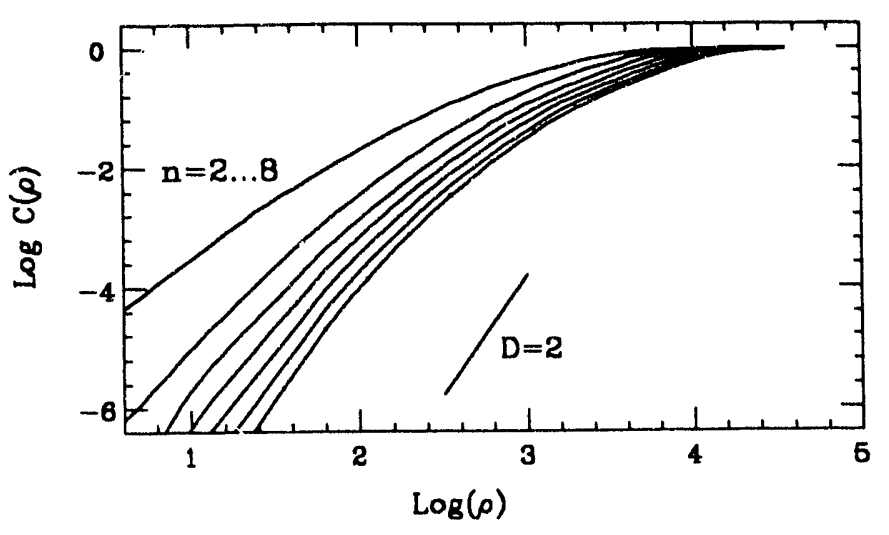

Fig. 4: Correlation data for a turn-by-turn BPM.

\section{Two or more degrecs of freedom}

With betatron coupling or chromaticity, the series $\langle x\rangle_{t}$ reflects a Hamiltonian with $D \geq 2$. Still it is possible to derive analytic solutions for the center-of-mass motion after injection but the number of parameters that have to be determined from data increases considerably. One way tc overcome this is to filter the data in the frequency domali and reduce the 2 or 3 degrees to one. The discussion here is limited to tests of calculations and the available data types.

\section{Higher dimensionality of different 'data' types}

An analysis of $\langle x\rangle_{t}$ from $3 \mathrm{D}$ tracking with DESPOT for the first 1000 turns implied a value consistent with $D \approx 1.5$ as though the different degrees of freedom were nearly uncoupled. The result for the energy was especially clean with $D=1$. At 1000 turns, coherent oscillations from injection errors in position and energy had nearly damped whereas at 500 turns $D \approx 2$ for $\langle x\rangle_{t}$.

A BPM monitor [5] that gives turn-by-turn data for an FFT has $D \geq 2$ as implied above. Fig. 4 shows an example for the first 1000 turns after injection when there is no betatron coupling. For a synchrotron light monitor (SLM) we expect a similar or higher $D$.

Fig's. 5\&6 show some results [6] for a damped beam that is first perturbed by an extraction kicker and then again by an injection kicker near turn 130 . The effects in $\mathrm{x}$ are more emphatic and a good example of a matched beam

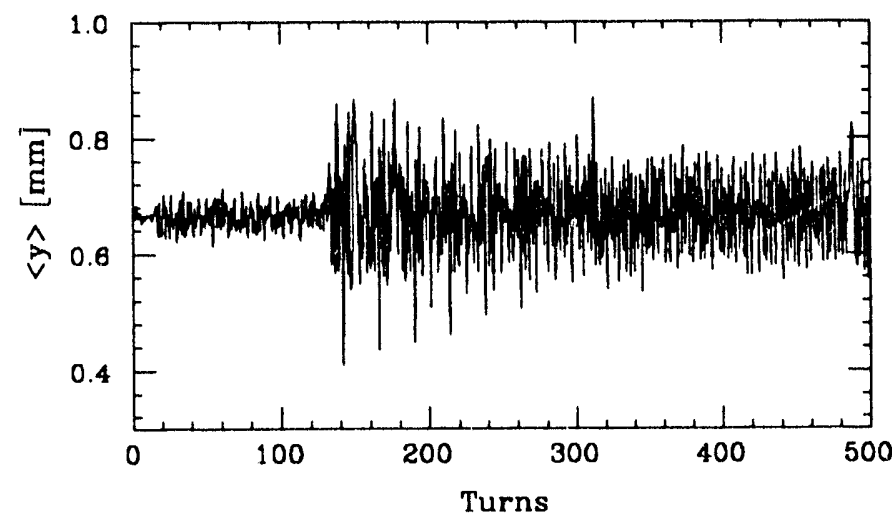

Fig. 5: (y) vs turns for kicked data from a SLM.

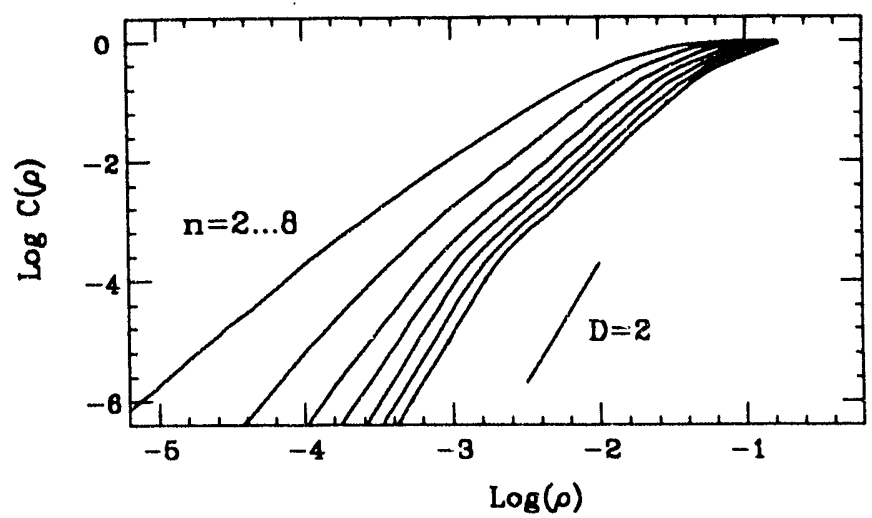

Fig. 6: Correlation data for a 'turn-by-turn' SLM. with $b=1$ that decoheres and filaments when deflected. Analysis of the 500 turns for $x$ give a result similar to Fig. 4 with $D \approx 2$, but the y data look totally different and appear noisy so that one expects a higher phase space dimensionality. The result for all 500 turns is shown in Fig. 6. The $D=1$ trend at the larger correlation lengths disappears when one analyzes only the turns for $N>130$ which may indicate coupling from the extraction kicker.

\section{Conclusions}

Dimensional analysis of real data show insignificant noise [7]. Decimating data, to filter, compress or match sampling capacity should extend the applicability. More SLM data for different orbits could study coupling from the kickers. The analysis is simple for $n<10$ so it should be interesting for many accelerator studies such as nonlinear resonances (i.e. optimum working points) or coupling in multi-bunch or flat beams (e.g. from the kickers) as well as the parameter dependence of stochastic effects in the beam-beam interaction or in long-term tracking.

\section{REFERENCES}

[1] J.E. Spencer, IEEE PAC (1991)1440. Dynamical models may be used to define and train neural nets.

[2] See Ref. [1] and Edward Ott, Celso Grebogi and James Yorke, Phys. Rev. Lett. 64(1990)1196. They might argue that because most attractors are chaotic, structural stability is mostly irrelevant and even an impediment.

[3] N.H.Packard, J.P.Crutchfield, J.D.Farmer and R.S. Shaw, Phys. Rev. Lett. 45(1980)712 and P. Grassberger and I. Procaccia, Phys. Rev. Lett. 50(1983)346. Alternative algorithms for on-line control will be discussed elsewhere.

[4] H. Moshammer, SLAC-PUB-6063. I thank this author and those below for many useful discussions.

[5] See R. Stege, K. Jobe and M. Ross, this conference.

[6] 'The data are M. Minty's. See SLAC-PUB-5993.

[7] The only other analysis for accelerator data we are aware of was Voelker Ziemann's. It showed large stochastic effects in linac BPM data but that data was necessarily taken differently. 

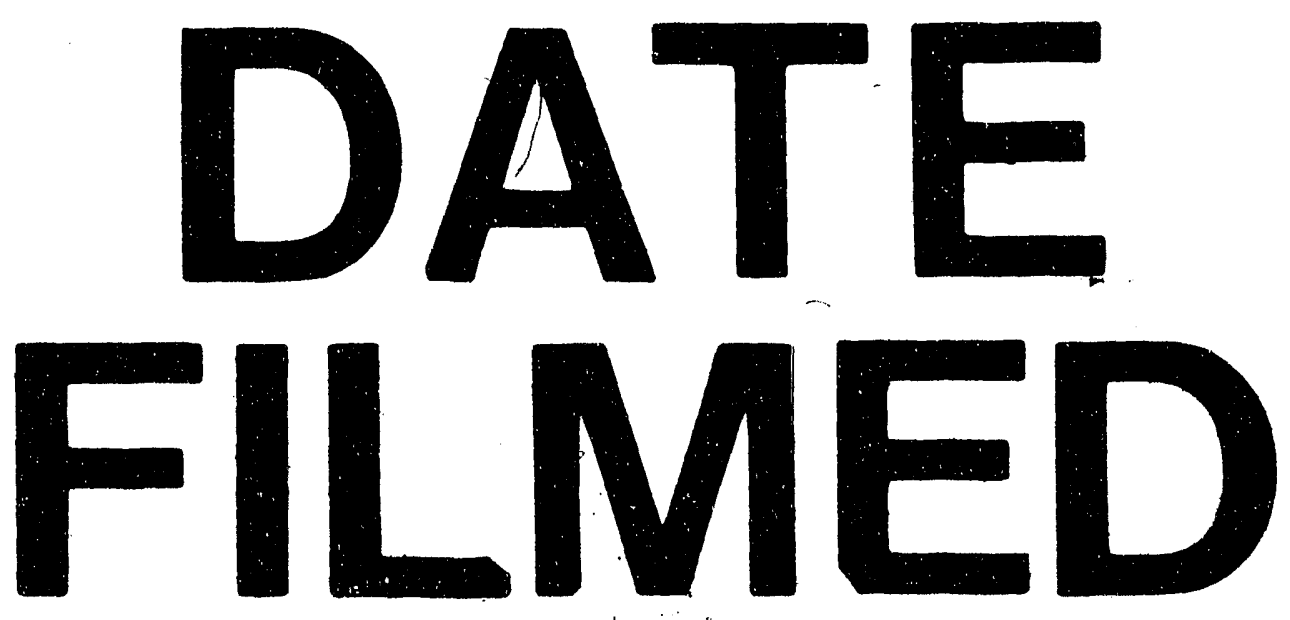

$\downarrow$

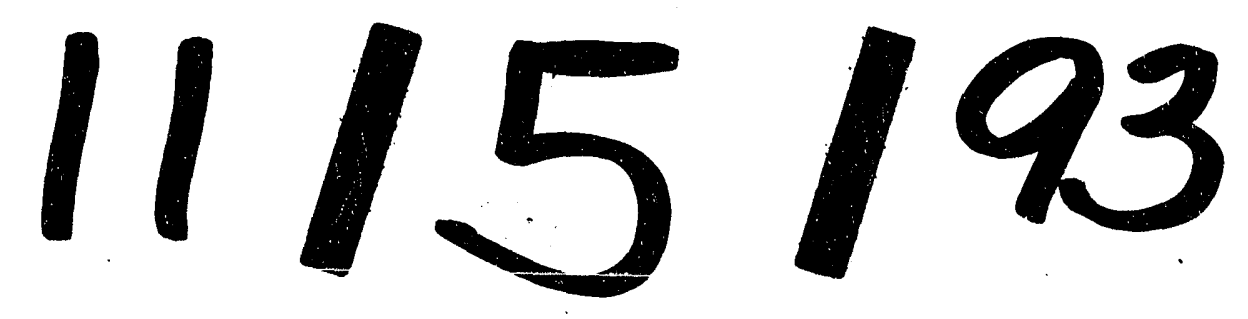


